\title{
RC-Graphs and Schubert Polynomials
}

\author{
Nantel Bergeron and Sara Billey
}

\section{CONTENTS}

1. Introduction

2. Background on Schubert Polynomials

3. Constructing Schubert Polynomials From RC-Graphs

4. Double Schubert Polynomials

5. Monk's Rule

6. Conjectures

Acknowledgements

References

Bergeron was supported by the National Science Foundation.

Billey was supported by the National Physical Science

Consortium, IBM and UCSD.

\begin{abstract}
Using a formula of Billey, Jockusch and Stanley, Fomin and Kirillov have introduced a new set of diagrams that encode the Schubert polynomials. We call these objects rc-graphs. We define and prove two variants of an algorithm for constructing the set of all rc-graphs for a given permutation. This construction makes many of the identities known for Schubert polynomials more apparent, and yields new ones. In particular, we give a new proof of Monk's rule using an insertion algorithm on rc-graphs. We conjecture two analogs of Pieri's rule for multiplying Schubert polynomials. We also extend the algorithm to generate the double Schubert polynomials.
\end{abstract}

\section{INTRODUCTION}

Schubert polynomials (defined in Section 2) are of interest in algebraic geometry and in combinatorics. Their history goes back to the the nineteenth century, but the modern notion is due to Lascoux and Schützenberger [1982; 1985], who developed a beautiful theory of these polynomials. A complete exposition can be found in [Macdonald 1991].

Our approach to computing Schubert polynomials is an algorithmic one. The idea is related to a conjecture originally due to Axel Kohnert [1990], saying that the Schubert polynomials could be constructed by applying a recursive algorithm on the diagram $D(w)$ defined from a permutation $w=$ $\left[w_{1}, \ldots, w_{n}\right]$ as the set of pairs $\left(i, w_{j}\right)$ for which $j>i$ and $w_{j}<w_{i}$. Each diagram that appears in the recursion contributes a term to the Schubert polynomial. At this time, Kohnert's conjecture has not been proved except in the special case that $w$ is a vexillary permutation (or 2143-avoiding). We have verified the conjecture for every permutation in the symmetric group $S_{7}$. 
In [Bergeron 1992] another algorithm, similar to Kohnert's, is given. This algorithm again starts with $D(w)$ but is computationally more complex. Furthermore, some identities about Schubert polynomials cannot readily be established using this method of computation. Therefore, we were driven to find yet another constructive method of computing Schubert polynomials from some other set of diagrams.

Fomin and Kirillov [1993] introduced a new set of diagrams that encode the Schubert polynomials. We call this object an rc-graph (for reducedword compatible sequence graph). In the spirit of Kohnert's conjecture, we are interested in constructing Schubert polynomials by doing "moves" on rc-graphs. We will define and prove two variants of an algorithm for constructing the set of all rcgraphs for a given permutation in Section 3. This algorithm has been much more efficient in time and space than previously known ones. It can also be extended to generate the double Schubert polynomials as well. Using this construction, many of the identities known for Schubert polynomials become more apparent and new identities have emerged. In particular, Section 5 gives a new proof of Monk's rule, using an insertion algorithm on rc-graphs.

Experimentation on computers has greatly facilitated our work. We have gained an invaluable amount of intuition about Schubert polynomials by looking at data, we have been able to rule out false conjectures quickly, and we have found two very interesting conjectures. In Section 6 , we conjecture two analogs of Pieri's rule for multiplying Schubert polynomials. We explain how we used computers to find these conjectures.

\section{BACKGROUND ON SCHUBERT POLYNOMIALS}

We will briefly review the basic terminology from [Macdonald 1991]. Let $S_{n}$ be the symmetric group on $n$ elements; we write a permutation $w \in S_{n}$ in one-line notation as $\left[w_{1}, w_{2}, \ldots, w_{n}\right]$, for $w_{1}, \ldots, w_{n}$ in $\{1,2, \ldots, n\}$. Let $s_{i}$ denote the transposition that interchanges the $i$-th and $(i+1)$-st entries when acting from the right on a permutation. It is well known that $s_{1}, \ldots, s_{n-1}$ generate $S_{n}$, with the relations

$$
\begin{aligned}
s_{i}^{2} & =1, \\
s_{i} s_{j} & =s_{j} s_{i} \quad \text { for }|i-j|>1, \\
s_{i} s_{i+1} s_{i} & =s_{i+1} s_{i} s_{i+1} .
\end{aligned}
$$

For $w \in S_{n}$, the length $l(w)$ of $w$ is the length of a shortest string $s_{a_{1}} s_{a_{2}} \ldots s_{a_{p}}$ representing $w$; we call the $p$-tuple $a_{1} a_{2} \ldots a_{p}$ a reduced word for $w$. Let $R(w)$ denote the set of all reduced words for a permutation $w$.

Let $\mathbb{Z}\left[x_{1}, x_{2}, \ldots, x_{n}\right]$ denote the ring of polynomials in $n$ variables with coefficients in $\mathbb{Z}$. We define an action of $w \in S_{n}$ on $f \in \mathbb{Z}\left[x_{1}, \ldots, x_{n}\right]$ by $w f\left(x_{1}, x_{2}, \ldots, x_{n}\right)=f\left(x_{w_{1}}, x_{w_{2}}, \ldots, x_{w_{n}}\right)$. From this we can define the divided difference operators

$$
\partial_{i} f\left(x_{1}, x_{2}, \ldots, x_{n}\right)=\frac{f\left(x_{1}, \ldots, x_{n}\right)-s_{i} f\left(x_{1}, \ldots, x_{n}\right)}{x_{i}-x_{i+1}}
$$

for $1 \leq i \leq n-1$. It is easy to check that $\partial_{i}^{2}=0$, $\partial_{i} \partial_{j}=\partial_{j} \partial_{i}$ if $|i-j|>1$ and $\partial_{i} \partial_{i+1} \partial_{i}=\partial_{i+1} \partial_{i} \partial_{i+1}$. Therefore $\partial_{w}=\partial_{a_{1}} \partial_{a_{2}} \ldots \partial_{a_{p}}$ does not depend on the choice of reduced word $a_{1} a_{2} \ldots a_{p} \in R(w)$. In addition, $\partial_{a_{1}} \partial_{a_{2}} \ldots \partial_{a_{p}}=0$ if $a_{1} a_{2} \ldots a_{p}$ is not reduced.

Definition. For each permutation $w \in S_{n}$, the $S c h u$ bert polynomial $\mathfrak{S}_{w}$ is

$$
\mathfrak{S}_{w}=\partial_{w^{-1} w_{0}} x_{1}^{n-1} x_{2}^{n-2} \ldots x_{n-1}^{1} x_{n}^{0},
$$

where $w_{0}=[n, n-1, \ldots, 1]$ is the longest element of $S_{n}$.

For $m>n$, we can associate with a permutation $w=\left[w_{1}, w_{2}, \ldots, w_{n}\right] \in S_{n}$ the permutation $v=$ $\left[w_{1}, w_{2}, \ldots, w_{n}, n+1, \ldots, m\right] \in S_{m}$. Now $v$ and $w$ have the same set of reduced words (the generators being interpreted in $S_{n}$ or $S_{m}$ as appropriate), so their length is also the same. From now on we identify two such permutations, making $S_{n}$ into a subgroup of $S_{m}$ for $n<m$. We set $S_{\infty}=\bigcup S_{n}$, and still write $w \in S_{\infty}$ as $w=\left[w_{1}, w_{2}, \ldots, w_{n}\right]$ if $w \in S_{n}$. The definition of a Schubert polynomial applies equally well to $w \in S_{\infty}$; it is not obvious at 
this point that the result is independent of $n$, but this will follow from Theorem 2.1.

The theory of Schubert polynomials is closely intertwined with the study of reduced words. The key to understanding this relationship is Theorem 2.1 , which amounts to an alternative definition of Schubert polynomials. This result was originally conjectured by Richard Stanley, and first proved in [Billey et al. 1993] and subsequently in [Fomin and Stanley 1991] in a very elegant way.

Definition. If $\mathbf{a}=a_{1} \ldots a_{p} \in R(w)$, we say that a $p$-tuple $\boldsymbol{\alpha}=\left(\alpha_{1}, \ldots, \alpha_{p}\right)$ of (strictly) positive integers is a-compatible if

$$
\begin{aligned}
& \alpha_{1} \leq \alpha_{2} \leq \cdots \leq \alpha_{p} \\
& \alpha_{j} \leq a_{j} \quad \text { for } 1 \leq j \leq p, \\
& \alpha_{j}<\alpha_{j+1} \quad \text { if } a_{j}<a_{j+1} .
\end{aligned}
$$

Let $C(\mathbf{a})$ denote the set of all a-compatible sequences.

Theorem 2.1 [Billey et al. 1993; Fomin and Stanley 1991]. For any permutation $w \in S_{\infty}$,

$$
\mathfrak{S}_{w}=\sum_{\mathbf{a} \in R(w)} \sum_{\alpha_{1} \ldots \alpha_{p} \in C(\mathbf{a})} x_{\alpha_{1}} x_{\alpha_{2}} \ldots x_{\alpha_{p}} .
$$

It is easy to compute a Schubert polynomial using this theorem, provided one can compute all reduced words. For example, for $w=[3,1,2,5,4]$ the reduced words are 214, 241 and 421. For each of these we compute all compatible sequences as follows:

$\begin{array}{lll}\frac{214}{112} & \underline{241} & \underline{421} \\ 113 & & \\ 114 & \end{array}$

Therefore $\mathfrak{S}_{[3,1,2,5,4]}=x_{1}^{3}+x_{1}^{2} x_{2}+x_{1}^{2} x_{3}+x_{1}^{2} x_{4}$.

\section{CONSTRUCTING SCHUBERT POLYNOMIALS FROM RC-GRAPHS}

In this section we define rc-graphs and an algorithm for computing Schubert polynomials. The goal of our algorithm is to start with a particular rc-graph and apply a sequence of transformations, thereby obtaining all rc-graphs for a permutation. After proving several lemmas, we state and prove Theorem 3.7, our main theorem, which states that this algorithm constructs the Schubert polynomials.

There are in fact two flavors of the algorithm, dual in a certain sense to one another. The duality follows from Lemma 3.2, which gives a correspondence between the rc-graphs of $w$ and those of $w^{-1}$.

We conclude this section with some corollaries that follow easily from Theorems 3.7.

Definition. Given a reduced word $\mathbf{a}=a_{1} a_{2} \ldots a_{p}$ and an a-compatible sequence $\boldsymbol{\alpha}=\alpha_{1} \alpha_{2} \ldots \alpha_{p}$, the reduced-word compatible sequence graph or rc-graph $(\mathbf{a}, \boldsymbol{\alpha})$ is the subset $D(\mathbf{a}, \boldsymbol{\alpha})$ of $\{1,2, \ldots\} \times\{1,2, \ldots\}$ consisting of pairs

$$
\left(\alpha_{k}, a_{k}-\alpha_{k}+1\right) \quad \text { for } 1 \leq k \leq p .
$$

We let $\mathcal{R C}(w)=\{D(\mathbf{a}, \boldsymbol{\alpha}): \mathbf{a} \in R(w), \boldsymbol{\alpha} \in C(\mathbf{a})\}$.

We will depict $D(\mathbf{a}, \boldsymbol{\alpha})$ as an incidence table in upper triangular form, with rows and columns labeled $1,2, \ldots$ For example, one reduced word for $[3,1,4,6,5,2]$ is $\mathbf{a}=521345$, and $\boldsymbol{\alpha}=111235$ is a-compatible. The rc-graph $D(\mathbf{a}, \boldsymbol{\alpha})$ is given by

$$
\begin{array}{lllllll} 
& 1 & 2 & 3 & 4 & 5 & 6 \\
1 & + & + & \cdot & \cdot & + & \cdot \\
2 & \cdot & + & \cdot & \cdot & \cdot & \\
3 & \cdot & + & \cdot & \cdot & & \\
4 & \cdot & \cdot & \cdot & & & \\
5 & + & \cdot & & & \\
6 & \cdot & & & &
\end{array}
$$

where $\mathrm{a}+$ represents a pair that belongs to the graph and $\mathrm{a} \cdot$ one that does not.

Remarks 3.1. It will soon become clear why it is more convenient in our context to use the pairs $\left(\alpha_{k}, a_{k}-\alpha_{k}+1\right)$ instead of the pairs $\left(\alpha_{k}, a_{k}\right)$.

One can also define rc-graphs in the general case when $\mathbf{a}$ is not a reduced word, but we will not need that. 
Given an rc-graph $D(\mathbf{a}, \alpha)$, one can recover a by reading out the numbers $j+i-1$ for the occupied positions $(i, j)$ from right to left in each row from top to bottom. The compatible sequence $\alpha$ is recovered by reading the row numbers of the occupied positions, in the same order. If $a_{1} a_{2} \ldots a_{p}$ is the reduced word read from the rc-graph $D$, we let

$$
\operatorname{perm} D=s_{a_{1}} s_{a_{2}} \ldots s_{a_{p}}
$$

be the permutation such that $D \in \mathcal{R C}(\operatorname{perm} D)$.

It follows from the definition of a compatible sequence that, if perm $D \in S_{n}$, all the elements $(i, j)$ of $D$ satisfy $i+j<n$. Conversely, any set of pairs of positive integers $(i, j)$ that lies in the area $i+j<n$ and that gives a reduced word $\mathbf{a}$ by means of the above reading is an rc-graph of a permutation of $S_{n}$, and the corresponding sequence of row numbers is a-compatible.

The rc-graphs originally introduced by Fomin and Kirillov [1993] are a bit more elaborate. They represent a planar history of the inversions of $w=$ perm $D$. To get from the definition above to this other one, we draw strands that cross at the positions $(i, j) \in D$ and avoid each other at the positions $(i, j) \notin D$. Here is the diagram for the rcgraph in (3.1):

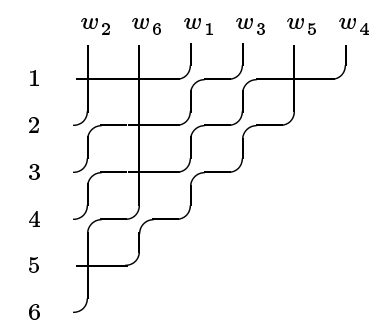

It is easy to see that, for $D \in \mathcal{R C}(w)$, the strand that starts at the top of the $w_{i}$-th column wends its way down to the $i$-th row on the left side of the diagram. No two strands can cross more than once, since by removing two such crossings we would obtain a shorter representation for the permutation $w$, contradicting the fact that the given word is reduced. Thus $\mathcal{R}(w)$ is the set of all such strand configurations with exactly $l(w)$ crossings. From now on we will often think of our rc-graphs as strand diagrams, but we will draw the strands only when needed. Moreover we will not draw the "sea" of wavy strands past the crossings with $i+j$ maximal.

Lemma 3.2. The transpose $D^{t}$ of an rc-graph $D$ in $\mathcal{R C}(w)$ is an rc-graph in $\mathcal{R C}\left(w^{-1}\right)$.

Proof. If $D \in \mathcal{R C}(w)$, the strands in $D^{t}$ trace out the permutation $w^{-1}$. Furthermore, $l(w)=l\left(w^{-1}\right)$. Therefore, the number of crossings of $D^{t}$ is minimal.

We also write $\rho(D)$ for $D^{t}$, thus defining a bijection $\rho: \mathcal{R C}(w) \rightarrow \mathcal{R C}\left(w^{-1}\right)$. Vic Reiner has suggested the same map using only reduced words and compatible sequences.

If we use the notation

$$
x_{D}=\prod_{(i, j) \in D} x_{i}
$$

the following corollary is a simple consequence of Theorem 2.1. This was also noted in [Fomin and Kirillov 1993].

Corollary 3.3. For any permutation $w \in S_{\infty}$,

$$
\mathfrak{S}_{w}=\sum_{D(\mathbf{a}, \boldsymbol{\alpha}) \in \mathcal{R} \mathcal{C}(w)} x_{D(\mathbf{a}, \boldsymbol{\alpha})}
$$

We now introduce moves on rc-graphs that are analogous to the moves in Kohnert's conjecture [Kohnert 1990].

For $w \in S_{\infty}$ and $D \in \mathcal{R C}(w)$, a ladder move $L_{i j}$ is a change of the following type:

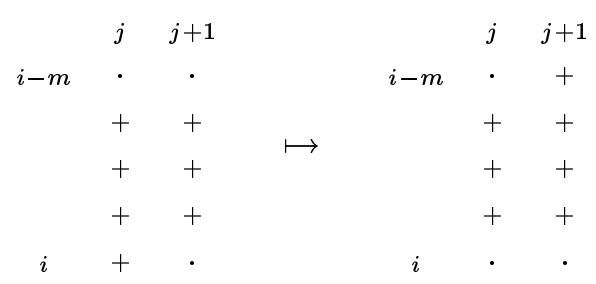

Here the two columns are adjacent and the number of rows involved is arbitrary. Formally, $L_{i j}(D)=$ $D \cup\{(i-m, j+1)\} \backslash\{(i, j)\}$, where the following conditions must be satisfied: 
- $(i, j) \in D,(i, j+1) \notin D$,

- $(i-m, j),(i-m, j+1) \notin D$ for some $0<m<i$, and

- $(i-k, j),(i-k, j+1) \in D$ for each $1 \leq k<m$.

A chute move $C_{i j}$ is a change of the following type (we leave the formal description to the reader):

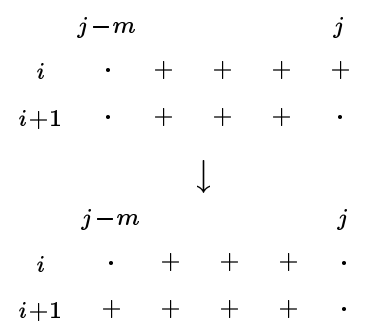

It is evident from these definitions and from Lemma 3.2 that:

Lemma 3.4. Transposition conjugates chute moves to ladder moves, and vice versa. In symbols,

$$
\rho\left(L_{i j}(D)\right)=C_{j i}(\rho(D))
$$

for any rc-graph $D$.

Lemma 3.5. Ladder and chute moves preserve the permutation associated with an rc-graph. In symbols, perm $C_{i j}(D)=\operatorname{perm} D$ if $D$ is an rc-graph to which $C_{i j}$ can be applied, and likewise for $L_{i j}$.

Proof. The strands in the region of a chute move look like this:

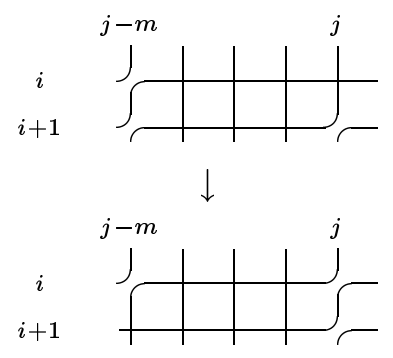

Clearly the move does not affect the way in which the strings are permuted. Transposition proves the case of a ladder move.

Next we give a criterion for an rc-graph to be the result of a chute move. Note that if the same chute move is applied to two different rc-graphs, the results are also different; therefore we can talk about inverse chute (and ladder) moves.

Lemma 3.6. An rc-graph $D \in \mathcal{R C}(w)$ is the result of a chute move-or, equivalently, it admits an inverse chute move - if and only if there is some pair $(i, j) \notin D$ such that $(i+1, j) \in D$.

Geometrically, the criterion for there not being an inverse chute move is that all the pluses in each column are clumped together at the top.

Proof. Suppose there is $(i, j) \notin D$ with $(i+1, j) \in$ $D$. Look right along row $i+1$ for the smallest $k>j$ such that $(i+1, k) \notin D$. There must be such a $k$, since $D$ contains only a finite number of points. The situation then is the following:

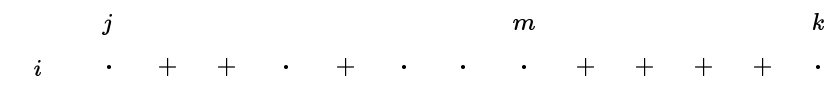

$$
\begin{aligned}
& i+1++\quad+\quad+\quad+\quad+\quad+\quad+\quad \text {. }
\end{aligned}
$$

where the positions on the top row strictly between $j$ and $k$ may be filled with + or $\cdot$, but the position $(i, k)$ must have a dot; if that position had a + we would have

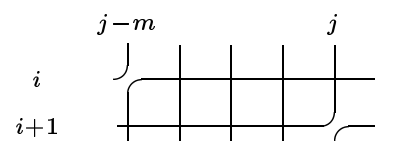

which is impossible because, as already remarked, two strands cannot cross twice. Now look to the left of $(i, k)$ for the largest $m<k$ such that $(i, m) \notin$ $D$. Then $(i+1, m)$ is able to move to $(i, k)$ by an inverse chute move.

The converse also follows from this analysis.

To state our main theorem, we introduce two rcgraphs for $w \in S_{\infty}$ that are extremal in an appropriate sense:

$$
D_{\text {bot }}(w)=\left\{(i, c): c \leq m_{i}\right\},
$$

where $m_{i}=\#\left\{j: j>i\right.$ and $\left.w_{j}<w_{i}\right\}$, arises from the largest reduced word in reverse lexicographic 
order and the largest compatible sequence for this word in ordinary lexicographic order; and

$$
D_{\text {top }}(w)=\left\{(c, j): c \leq n_{j}\right\},
$$

where $n_{j}=\#\left\{i: i<w_{j}^{-1}\right.$ and $\left.w_{i}>j\right\}$, arises from the smallest reduced word in reverse lexicographic order and the smallest compatible sequence for this word in ordinary lexicographic order. We have $D_{\text {top }}(w)=D_{\text {bot }}^{t}\left(w^{-1}\right)$. Here are $D_{\text {top }}$ and $D_{\text {bot }}$ for the permutation of (3.1) on page 259:

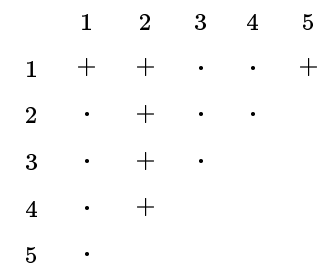

$$
\begin{array}{llllll} 
& 1 & 2 & 3 & 4 & 5 \\
1 & + & + & \cdot & \cdot & \cdot \\
2 & \cdot & \cdot & \cdot & \cdot & \\
3 & + & \cdot & \cdot & & \\
4 & + & + & & & \\
5 & + & & & &
\end{array}
$$

Let $\mathcal{C}(D)$ be the set of rc-graphs that can be derived from $D$ by some sequence of (forward) chute moves. Define $\mathcal{L}(D)$ analogously, with "ladder" replacing "chute".

Theorem 3.7. Let $w \in S_{\infty}$.

(a) $D_{\text {top }}(w)$ does not admit an inverse chute.

(b) Any element of $\mathcal{R C}(w)$ other than $D_{\text {top }}(w)$ admits an inverse chute.

(c) $\mathcal{C}\left(D_{\text {top }}(w)\right)=\mathcal{R} \mathcal{C}(w)=\mathcal{L}\left(D_{\text {bot }}(w)\right)$.

(d) $\mathfrak{S}_{w}=\sum_{D \in \mathcal{C}\left(D_{\mathrm{top}}(w)\right)} x_{D}=\sum_{D \in \mathcal{L}\left(D_{\mathrm{bot}}(w)\right)} x_{D}$.

Proof. (a) follows from Lemma 3.6, since by definition every column of $D_{\text {top }}(w)$ has an initial run of pluses and no others.

(b) Suppose that $w \in S_{n}$. Consider, for all $w^{\prime} \in$ $S_{n}$, all rc-graphs $D \in \mathcal{R C}\left(w^{\prime}\right)$ that don't admit inverse chutes. For such a graph, column $j$ has between 0 and $n-j$ pluses clumped at the top, again by Lemma 3.6. This gives $n$ choices for the first column, $n-1$ for the second, and so on, so there are at most $n$ ! such graphs. By part (a), there is at least one such graph, $D_{\text {top }}\left(w^{\prime}\right)$, for each permutation. Therefore all graphs of this type are of the form $D_{\text {top }}\left(w^{\prime}\right)$. (c) Anything obtained from $D_{\text {top }}$ by chute moves is in $\mathcal{R C}(w)$, by Lemma 3.5. Now an inverse chute move pushes up a + , so any graph $D \in \mathcal{R C}(w)$ must turn after finitely many inverse chutes into one that does not admit an inverse chute - that is, into $D_{\text {top }}(w)$, according to part (b). Reversing this sequence and applying chutes to $D_{\text {top }}(w)$ we recover $D$. This proves the first equality. The second follows from the first by duality: more precisely,

$$
\begin{aligned}
\mathcal{R C}(w) & =\rho\left(\mathcal{R C}\left(w^{-1}\right)\right)=\rho\left(\mathcal{C}\left(D_{\mathrm{top}}\left(w^{-1}\right)\right)\right) \\
& =\rho\left(\mathcal{C}\left(\rho\left(D_{\mathrm{bot}}(w)\right)\right)\right)=\mathcal{L}\left(D_{\mathrm{bot}}(w)\right),
\end{aligned}
$$

by Lemmas 3.2 and 3.4 .

(d) follows from (c) and Corollary 3.3.

As an example, Figure 1 shows the computation of $\mathfrak{S}_{[1432]}$ using ladder moves.

Chute and ladder moves define two partial orders on $\mathcal{R} \mathcal{C}(w)$, with relations $L_{i j}(D) \prec D$ and $C_{i j}(D) \prec D$, respectively. These partial orders are dual to one another.

The following result restricts the relations among the elements in the partial ordered set. However, there are still multiple paths to some of the rcgraphs.

Corollary 3.8. We can generate $\mathcal{R C}(w)$ using only chute moves $C_{i j}$ such that $i$ is the largest in column $j$, that is, those satisfying $(k, j) \notin D$ for all $k>j$. Similarly, we can generate $\mathcal{R C}(w)$ using only rightmost ladder moves $L_{i j}$, that is, those satisfying $(i, k) \notin D$ for all $k>i$.

Proof. Given $D \in \mathcal{R C}(w)$ different from $D_{\text {top }}(w)$, choose an inverse chute move $C_{i j}^{-1}$ such that $i$ is as large as possible. The point $(i, j)$ must be the lowest point in column $j$ of $C_{i j}^{-1}(D)$, otherwise there exists a point $(k, j) \notin D$ with $(k+1, j) \in D$, and hence another possible inverse chute move $C_{k j}^{-1}$ with $k>i$. Next choose the lowest inverse chute move possible on $C_{i j}^{-1}(D)$. Continue applying the lowest move until there are no inverse chute moves possible. Reversing this sequence gives a sequence of chute moves $C_{i j}$ such that $(i, j)$ is the lowest point in column $i$ that transforms $D_{\text {top }}(w)$ to $D$. 


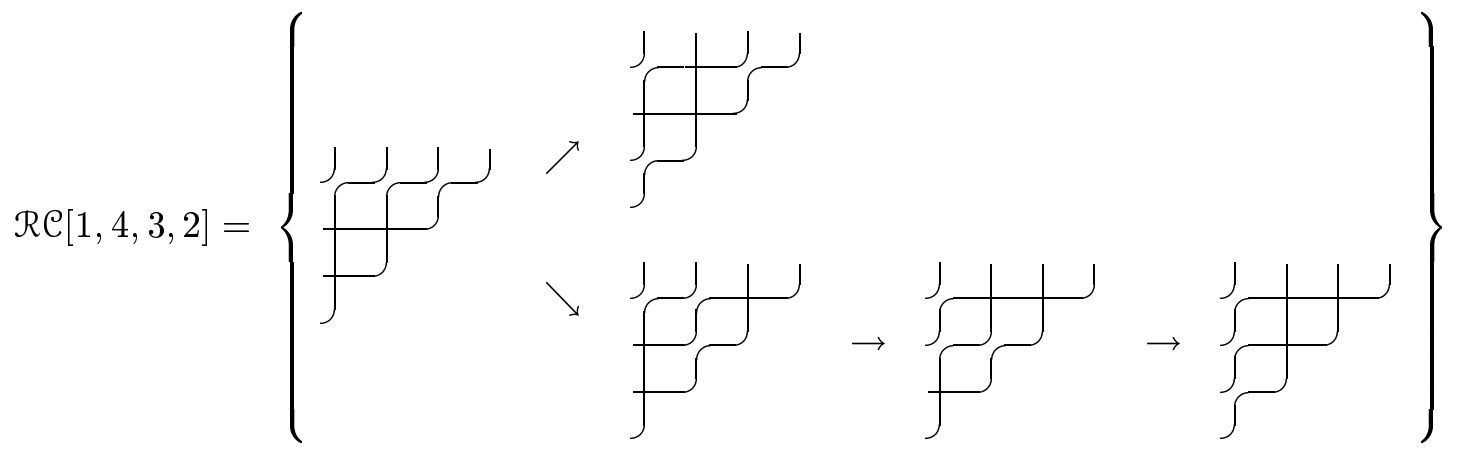

FIGURE 1. Computation of the Schubert polynomial of $w=[1,4,3,2]$ using Theorem 3.7. Starting from $D_{\text {bot }}(w)$ (leftmost diagram) we apply all possible ladder moves and compute the monomial for each graph so obtained. The result is $\mathfrak{S}_{[1432]}=x_{2}^{2} x_{3}+x_{1} x_{2}^{2}+x_{1} x_{2} x_{3}+x_{1}^{2} x_{3}+x_{1}^{2} x_{2}$.

Transposing this proof, we get the result for ladder moves.

Algebraic proofs of Corollaries 3.9, 3.10, and 3.12 appear in [Macdonald 1991]. Corollary 3.11 first appeared in [Billey et al. 1993].

Corollary 3.9. The Schubert polynomials, indexed by permutations in $S_{\infty}$, form an integral basis for

$$
\mathbb{Z}\left[x_{1}, x_{2}, \ldots\right] \text {. }
$$

Proof. The leading term of each $\mathfrak{S}_{w}$, in reverse lexicographic order, is given by $D_{\text {bot }}(w)$. Each $D_{\text {bot }}$ is unique, so each leading term is unique. Furthermore, given any monomial $x_{1}^{\alpha_{1}} \ldots x_{m}^{\alpha_{m}}$, there exists a permutation $w$ such that $x_{D_{\mathrm{bot}}(w)}=x_{1}^{\alpha_{1}} \ldots x_{m}^{\alpha_{m}}$ : simply put $\alpha_{1}$ pluses at the beginning of row $1, \alpha_{2}$ on row 2 , and so on.

Corollary 3.10. Given permutations $u \in S_{m}$ and $v \in$ $S_{n}$, let $u \times v=\left[u_{1}, \ldots, u_{m}, v_{1}+m, \ldots, v_{n}+m\right]$ and $1_{m} \times v=\left[1, \ldots, m, v_{1}+m, \ldots, v_{n}+m\right]$. Then

$$
\mathfrak{S}_{u} \mathfrak{S}_{1_{m} \times v}=\mathfrak{S}_{u \times v} .
$$

Proof. Every rc-graph in $\mathcal{R C}(u)$ is contained in $\{(i, j): i+j<m\}$, and no rc-graph in $\mathcal{R C}\left(1_{m} \times v\right)$ contains points in $\{(i, j): i+j \leq m\}$. No rcgraph in $\mathcal{R}(u \times v)$ contains a point on the line $i+j=m$. Therefore, there is a bijection between $\mathcal{R C}(u) \times \mathcal{R C}\left(1_{m} \times v\right)$ and $\mathcal{R C}(u \times v)$, given by sending $\left(D_{1}, D_{2}\right)$ to $D_{1} \cup D_{2}$.
Let $\downarrow$ denote the operation inverse to $v \mapsto 1_{1} \times v$, in the notation of the preceding lemma, so that $\downarrow v=\left[v_{2}, v_{3}, \ldots, v_{n}\right]$ if $v \in S_{n}$ with $v_{1}=1$ (and $\downarrow v$ is undefined if $v_{1} \neq 1$ ).

Corollary 3.11. For $w \in S_{\infty}$, we have

$$
\mathfrak{S}_{w}\left(x_{1}, x_{2}, \ldots\right)=\sum x_{1}^{l(v)} \mathfrak{S}_{\downarrow v w}\left(x_{2}, x_{3}, \ldots\right),
$$

where the sum is over all permutations $v \in S_{\infty}$ such that $l(w)=l(v w)+l(v), v=s_{i_{1}} s_{i_{2}} \ldots s_{i_{p}}$ with $i_{1}<i_{2}<\ldots<i_{p}$, and $(v w)_{1}=1$.

Proof. There is a bijection

$$
\mathcal{R C}(w) \leftrightarrow \bigcup(v, \mathcal{R C}(\downarrow v w)),
$$

where the union is over all permutations $v \in S_{n}$ such that $l(w)=l(v w)+l(v), v=s_{i_{1}} s_{i_{2}} \ldots s_{i_{p}}$ with $i_{1}<i_{2}<\cdots<i_{p}$, and $(v w)_{1}=1$. The bijection is given by sending $D \in \mathcal{R C}(w)$ to $\left(v, D^{\prime}\right)$ if $v=s_{i_{1}} s_{i_{2}} \ldots s_{i_{p}}$, where the first row of $D$ are points in columns $i_{1}, i_{2}, \ldots, i_{p}$, and $D^{\prime}$ is the rcgraph obtained by removing the first row of $D$.

The next result is a generalization of Corollary 3.11.

Corollary 3.12. For any fixed positive integer $m$ and any $w \in S_{n}$, we have the decomposition

$$
\begin{aligned}
\mathfrak{S}_{w}\left(x_{1}, \ldots, x_{n}\right) & \\
& =\sum d_{u v}^{w} \mathfrak{S}_{u}\left(x_{1}, \ldots, x_{m}\right) \mathfrak{S}_{v}\left(x_{m+1}, \ldots, x_{n}\right),
\end{aligned}
$$

where the $d_{u v}^{w}$ are non-negative integers. 
Proof. Given a polynomial $f\left(x_{1}, x_{2}, \ldots, x_{m}\right)$, let $\rho_{m} f=f\left(x_{1}, \ldots, x_{m}, 0,0, \ldots\right)$. By an abuse of notation, we also let $\rho_{m}(\mathcal{R C}(w))=\{D \in \mathcal{R C}(w)$ : $\left.\rho_{m}\left(x_{D}\right)=x_{D}\right\}$. For each $w \in S_{\infty}$ and each $m$, there exists a bijection

$$
\mathcal{R C}(w) \leftrightarrow \bigcup \rho_{m}(\mathcal{R C}(u)) \times \mathcal{R C}(v),
$$

where the union is over all permutations $u, v$ such that $l(u)+l(v)=l(w)$ and $1_{m} \times v=u^{-1} w$. The bijection takes $D$ to

$$
\{(i, j) \in D: j \leq m\} \times\{(i, j-m):(i, j) \in D, j>m\} .
$$

Therefore,

$$
\begin{aligned}
& \mathfrak{S}_{w}\left(x_{1}, \ldots, x_{n}\right) \\
& =\sum_{\substack{l(u)+l(v)=l(w) \\
1_{m} \times v=u^{-1} w}}\left(\rho_{m} \mathfrak{S}_{u}\left(x_{1}, \ldots, x_{n}\right)\right) \mathfrak{S}_{v}\left(x_{m+1}, \ldots, x_{n}\right) .
\end{aligned}
$$

Finally, we can expand $\rho_{m} \mathfrak{S}_{u}$ in a positive sum of Schubert polynomials by the transition equation (4.16) of [Macdonald 1991].

\section{DOUBLE SCHUBERT POLYNOMIALS}

Double Schubert polynomials generalize the usual Schubert polynomials in two alphabets. The original definition, given by Lascoux and Schützenberger, was written in terms of divided difference operators. Our definition follows from [Macdonald 1991, eq. (6.3)]. We show that the double Schubert polynomials can also be represented graphically and that these graphs can be constructed by means of ladder moves on an initial graph.

Definition. We take two alphabets $X=\left\{x_{1}, x_{2}, \ldots\right\}$ and $Y=\left\{y_{1}, y_{2}, \ldots\right\}$. For $w \in S_{\infty}$, we define the double Schubert polynomial $\mathfrak{S}_{w}(X, Y)$ as

$$
\mathfrak{S}_{w}(X, Y)=\sum_{\substack{v^{-1} u=w \\ l(u)+l(v)=l(w)}}(-1)^{l(v)} \mathfrak{S}_{u}(X) \mathfrak{S}_{v}(Y) .
$$

Definition. A double rc-graph $E$ for a permutation $w$ consists of the following data: a pair $(u, v)$ of permutations such that $v^{-1} u=w$ and $l(w)=$ $l(u)+l(v)$, and an $r c$-graph for each of $u$ and $v$.
We usually think of the $r c$-graph for $v$ as being placed upside down above that of $u$, and label its rows with negative numbers. For this reason we call the rc-graph for $v$ the upper half of $E$, and the $r c$-graph for $u$ the lower half. For example, for $w=[4,3,2,1]$, one possible double rc-graph is

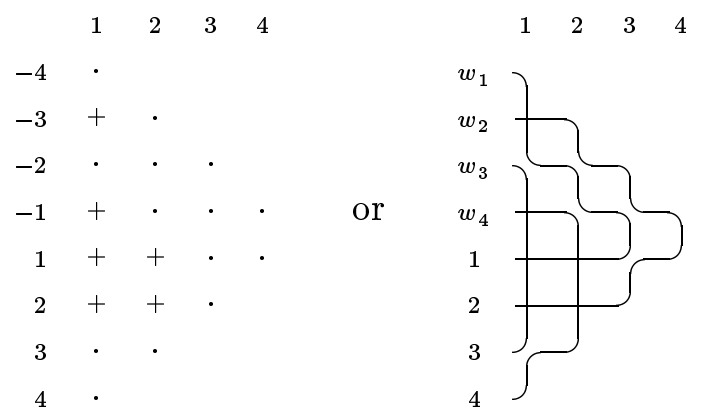

depending on whether or not we draw the strands. Here $u=[3,4,1,2]$ and $v=[2,1,4,3]$, and the lower and upper halves are

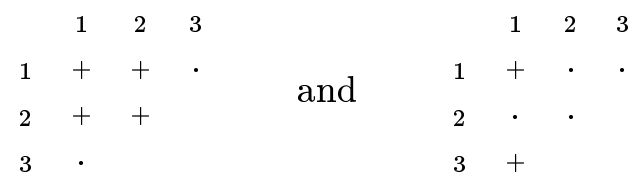

No two strands of a double rc-graph can cross twice. Two crossings in the same half are forbidden by the analogous result for simple rc-graphs, while one crossing in the upper half and one in the lower half would violate the condition $l(w)=l(u)+l(v)$, as their removal would yield a shorter representation for $w$.

There is a natural bijection between the double rc-graphs of $w$ and those of $w^{-1}$, given by interchanging the roles of $u$ and $v$ such that $w=v^{-1} u$, and the respective halves. Graphically, this corresponds to reflecting across $i=0$.

We denote the set of all double rc-graphs for $w$ by $\widetilde{\mathcal{R C}}(w)$. Given a double rc-graph $E$ with upper half $D_{-}$and lower half $D_{+}$, we define an associated monomial

$(x y)_{E}=(-1)^{\operatorname{card} D_{-}} x_{D_{+}} y_{D_{-}}=\prod_{(i, j) \in D_{+}} x_{i} \prod_{(i, j) \in D_{-}}-y_{i}$. 
Corollary 3.3 has the following counterpart:

$$
\mathfrak{S}_{w}(X, Y)=\sum_{E \in \widetilde{\mathcal{R C}}(w)}(x y)_{E}
$$

Indeed, by the definition of double Schubert polynomials and Corollary 3.3 we have

$$
\mathfrak{S}_{w}(X, Y)=\sum_{\substack{v^{-1} u=w \\ l(u)+l(v)=l(w)}} \sum_{C \in \mathcal{R} \mathcal{C}(u)} \sum_{D \in \mathcal{R} \mathcal{C}(v)}(-1)^{l(v)} x_{C} y_{D}
$$

and this latter sum clearly equals $\sum_{E \in \widetilde{\mathcal{R C}}}(x y)_{E}$.

We can extend the definition of a ladder or chute move to double rc-graphs. We will study ladder moves only and leave chutes to the reader. A ladder move on a double rc-graph $E$ composed of an upper half $D_{-}$and a lower half $D_{+}$is of one of three types:

(a) A (forward) ladder move in $D_{+}$.

(b) An inverse ladder move in $D_{-}$(note that since the upper half is drawn upside down, this type of ladder move still moves crossings up).

(c) A move of the form

$\begin{array}{rcccrcc} & j & j+1 & & & j & j+1 \\ -1 & \cdot & \cdot & & -1 & + & \cdot \\ 1 & + & + & & 1 & + & + \\ & + & + & \mapsto & & + & + \\ & + & + & & & + & + \\ & + & + & & & . & .\end{array}$

We denote by $\mathcal{L}(E)$ be the set of double rc-graphs that can be obtained from $E$ by ladder moves. As in the case of simple rc-graphs, ladder moves preserve the permutation $w$; this follows from Lemma 3.5 for moves of types (a) and (b), and from the following picture for moves of type (c):

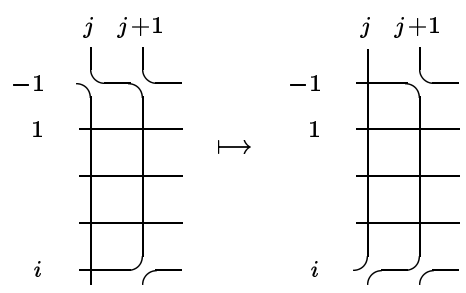

Note, however, that the permutations $v$ and $u$ of the upper and lower halves are not preserved in this case.

Let $E_{\mathrm{bot}}(w)$ be the double rc-graph for $w$ whose lower half is $D_{\text {bot }}(w)$ and whose upper half is trivial (no crossings).

Theorem 4.1. Let $w \in S_{\infty}$. Then

$$
\widetilde{\mathcal{R C}}(w)=\mathcal{L}\left(E_{\text {top }}(w)\right)
$$

and

$$
\mathfrak{S}_{w}(X, Y)=\sum_{E \in \mathcal{L}\left(E_{\mathrm{bot}}(w)\right)}(x y)_{E} .
$$

Stanley first noted that double Schubert polynomials could be expressed in terms of generalized compatible sequences as in Theorem 2.1.

Proof. The second equation follows from the first and from (4.1). To prove the first equation, we reason much as in the proof of Theorem 3.7. First, as already observed, any double rc-graph obtained from $E_{\text {bot }}(w)$ by ladder moves is in $\widetilde{\mathcal{R C}}(w)$.

To show, conversely, that any $E \in \widetilde{\mathcal{R C}}(w)$ is in $\mathcal{L}\left(E_{\text {bot }}(w)\right)$, we proceed by induction on the number of crossings in the upper half $D_{-}$of $E$. If $D_{-}$has no crossings, $E$ is in $\mathcal{L}\left(E_{\text {bot }}(w)\right)$, by Theorem 3.7(c) applied to the lower half $D_{+}$of $E$. Now suppose $D_{-}$has at least one crossing. Again using Theorem 3.7 on $D_{+}$, we can assume that $D_{+}=D_{\text {bot }}(u)$ for some permutation $u$. Using upside down ladder moves on $D_{-}$we may also assume that the row $i=-1$ is not empty.

Therefore we can find $j>0$ such that $(-1, j) \in$ $E$ and $(-1, j+1) \notin E$, then $i>0$ such that $(i, j) \notin E$ and $(k, j) \in E$ for $0<k<i$. Since $D_{+}=$ $D_{\text {bot }}(u)$, we must have $(i, j+1) \notin E$. We claim that we are allowed to move $(-1, j)$ to $(i, j)$ by an inverse ladder move of type (c). We need only check that $(k, j+1) \in E$ for all $0<k<i$. If we assume this is not the case, we would have the configuration shown on the right, for $k>0$ minimal with $(k, j+1) \notin$ $E$. This is impossible, since no two 
strands can cross twice. Hence, by inverse ladder moves we can decrease the number of crossings in $D_{-}$. By the induction hypothesis it follows that the new graph can be turned into $E_{\text {bot }}(w)$ with a sequence of inverse ladder moves. This concludes the proof.

From Theorem 4.1 one can check that $\mathfrak{S}_{w}(X, 0)=$ $\mathfrak{S}_{w}(X)$.

\section{MONK'S RULE}

In this section we give a new and elegant proof of Monk's rule [Macdonald 1991, eq. $\left(4.15^{\prime \prime}\right)$ ] for expanding $\mathfrak{S}_{w} \mathfrak{S}_{s_{r}}$ in the basis of Schubert polynomials. (Recall that $s_{r}$ is the $r$-th simple transposition.) The proof follows from an insertion algorithm for adding a crossing to an rc-graph in such a way that it produces another rc-graph. From this insertion algorithm, we were inspired to conjecture a rule for multiplying Schubert polynomials in a special case (Section 6).

We start with a simple observation: if we have the local configuration

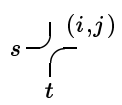

in position $(i, j)$ of an rc-graph, where $s$ and $t$ are the strand labels, then $s<t$ if and only if these two strands do not cross anywhere below this point (that is, on a row with $i^{\prime}>i$ ). This is clear, because strands are labeled by the row where they originate on the left, and they can cross at most once.
Algorithm 5.1 (Insertion). Given $D \in \mathcal{R C}(w)$ (where $\left.w \in S_{\infty}\right)$ and integers $r$ and $i$ with $r \geq i>0$, we produce a triple $I_{r}(D, i)=\left(D^{\prime}, k, l\right)$, where $k$ and $l$ are positive integers, $D^{\prime}$ is an rc-graph for a permutation of length $l(w)+1$, and $x_{D^{\prime}}=x_{D} x_{i}$. Since the leftmost strand on row $i$ is labeled $i \leq r$, there exists $j$ such that the position $(i, j)$ looks like

$$
\overbrace{t}^{s} \text { with } s \leq r<t
$$

We find $I_{r}(D, i)$ as follows (see also Figure 2):

Set $i_{0}=i$. Let $j_{0}$ be maximal such that the configuration at $\left(i_{0}, j_{0}\right)$ is as in (5.1). Add the crossing $\left(i_{0}, j_{0}\right)$ to $D$, and let $s_{0}=s$ and $t_{0}=t$ be the strands that cross there. If the result is an rc-graph, stop. This happens if the strands $s_{0}$ and $t_{0}$ do not cross again elsewhere. If, on the contrary, they also cross at $\left(i_{1}, j_{1}^{\prime}\right)$, delete this second crossing from $D$. Since $s_{0}<t_{0}$, we must have $i_{1}<i_{0}$, by the observation preceding the algorithm. Next, find $j_{1}<j_{1}^{\prime}$ maximal such that the configuration at $\left(i_{1}, j_{1}\right)$ is as in (5.1). Such a $j_{1}$ must exist since $i_{1}<i_{0} \leq r$. Add the crossing $\left(i_{1}, j_{1}\right)$ to $D$, and let $s_{1}$ and $t_{1}$ be the strands that cross there. If the result is an rc-graph, stop. Otherwise continue deleting and inserting crossings in the manner just explained. This process will eventually stop because the row numbers are strictly decreasing. If $p$ is the last step of the process, set $k=s_{p}$ and $l=t_{p}$, and let $D^{\prime}$ be the resulting graph, obtained from the original $D$ after $p$ add/delete steps and a final addition step.
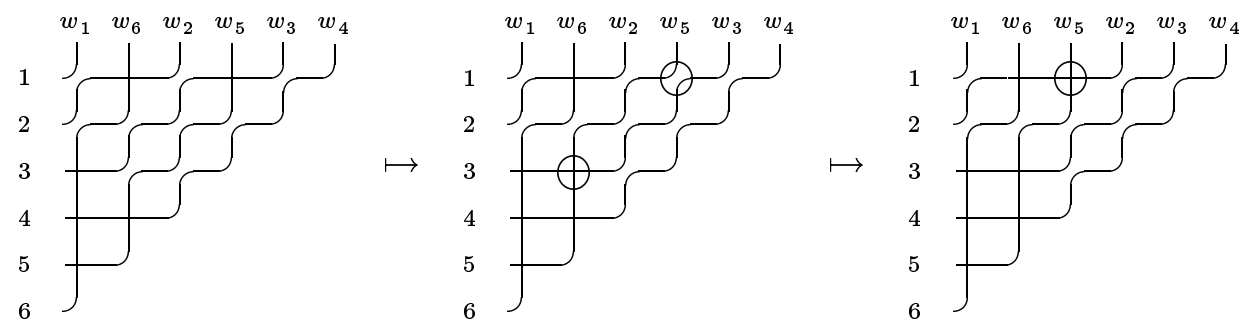

FIGURE 2. Application of the Insertion Algorithm 5.1 to an $r c$-graph $D \in \mathcal{R C}[1,3,5,6,4,2]$. Here $r=4$ and $i=3$. The insertion path is $(3,2),(1,4),(1,3)$. 
We call the sequence of positions

$$
\left(i_{0}, j_{0}\right),\left(i_{1}, j_{1}^{\prime}\right),\left(i_{1}, j_{1}\right), \ldots,\left(i_{p}, j_{p}^{\prime}\right),\left(i_{p}, j_{p}\right)
$$

the insertion path. The coordinates of an insertion path must satisfy the following inequalities:

$$
\begin{gathered}
i=i_{0}>i_{1}>\cdots>i_{p} \geq 1, \\
j_{0}<j_{1}^{\prime}>j_{1}<\cdots<j_{p}^{\prime}>j_{p} .
\end{gathered}
$$

Let $t_{k l}$ be the transposition that interchanges the elements in positions $k$ and $l$ when acting from the right on a permutation. Then $l\left(w t_{k l}\right)=l(w)+1$ when $k<l, w_{k}<w_{l}$, and for no index $m$ between $k$ and $l$ is there a $w_{m}$ such that $w_{k}<w_{m}<w_{l}$. Note that the output $\left(D^{\prime}, k, l\right)$ of the insertion algorithm satisfies perm $D^{\prime}=($ perm $D) t_{k l}$. Indeed, after each add/delete step, the resulting graph is still in $\mathcal{R C}(\operatorname{perm} D)$, since the added and deleted crossings involve the same pair of strands. The final step, when $\left(i_{p}, j_{p}\right)$ is added, involves the strands numbered $k=s_{p}$ and $l=t_{p}$, so it corresponds to multiplying the permutation on the right by $t_{k l}$. In addition, $l\left(\right.$ perm $\left.D^{\prime}\right)=l($ perm $D)+1$, since $D^{\prime}$ is an rc-graph by construction, and it has one more crossing than $D$.

Algorithm 5.2 (Reverse Insertion). Given a permutation $w$, integers $l>r \geq k>0$ with $l\left(w t_{k l}\right)=$ $l(w)+1$, and and rc-graph $D^{\prime} \in \mathcal{R C}\left(w t_{k l}\right)$, we can reverse the insertion algorithm to find a pair $(D, i)$, where $D \in \mathcal{R C}(w), i \leq r$, and $\left(D^{\prime}, k, l\right)=I_{r}(D, i)$. This is done as follows:

There is a unique position $\left(i_{0}, j_{0}\right) \in D^{\prime}$ where the strands $k$ and $l$ cross. Delete that crossing from $D^{\prime}$. Now if there is no position to the right of $\left(i_{0}, j_{0}\right)$ where the configuration is

$$
{ }_{s}^{t} \Gamma_{s} \quad \text { with } s \leq r<t
$$

stop. Otherwise, let $\left(i_{0}, j_{0}^{\prime}\right)$ be the position minimizing $j_{0}^{\prime}>j_{0}$ and having the configuration (5.2). Let $s_{1}=s$ and $t_{1}=t$ be the strands there. By the observation preceding Algorithm 5.1, these strands $s_{1}$ and $t_{1}$ must cross at some point $\left(i_{1}, j_{1}\right)$ where $i_{1}>i_{0}$ and $j_{1}<j_{0}^{\prime}$. Add $\left(i_{0}, j_{0}^{\prime}\right)$ from $D^{\prime}$, and delete $\left(i_{1}, j_{1}\right)$. If possible, find $\left(i_{1}, j_{1}^{\prime}\right)$ satisfying (5.2) and such that $j_{1}^{\prime}>j_{1}$ is minimal, and continue in this way. All strands below row $r$ have labels larger than $r$ and $i_{0}<i_{1}<\ldots<i_{p} \leq r$, so this process will eventually end. The last row $i_{p}$ from which we remove a point becomes $i$, and the modified rc-graph $D^{\prime}$ becomes $D$.

Theorem 5.3 (Monk's Rule). Given $w \in S_{\infty}$ and $a$ simple transposition $s_{r}$, we have

$$
\mathfrak{S}_{s_{r}} \mathfrak{S}_{w}=\sum_{\substack{k \leq r<l \\ l\left(w t_{k l}\right)=l(w)+1}} \mathfrak{S}_{w t_{k l}} .
$$

Proof. There is a monomial-preserving bijection

$$
\mathcal{R C}(w) \times \mathcal{R C}\left(s_{r}\right) \rightarrow \bigcup \mathcal{R C}\left(w^{\prime}\right),
$$

where the union is over all permutations $w^{\prime}=w t_{k l}$ such that $k \leq r<l$ and $l\left(w t_{k l}\right)=l(w)+1$. The bijection is given by $(D, i) \mapsto I_{r}(D, i)$, where we are using the fact that any rc-graph for $s_{r}$ contains a single crossing, on some row $i \leq r$, to identify $\mathcal{R e}\left(s_{r}\right)$ with $\{1, \ldots, r\}$.

The discussion immediately before Algorithm 5.2 shows that, if $D \in \mathcal{R C}(w)$, then $I_{r}(D, i) \in \mathcal{R C}\left(w^{\prime}\right)$ for some $w^{\prime}=w t_{k l}$ such that $k \leq r<l$ and $l\left(w t_{k l}\right)=l(w)+1$.

Conversely, assume that we are given an rc-graph $D^{\prime} \in \mathcal{R C}\left(w^{\prime}\right)$ where $w^{\prime}=w t_{k l}$ and $k \leq r<l$ and $l\left(w t_{k l}\right)=l(w)+1$. Each $D_{l}^{\prime}$ for $0 \leq l \leq p$ in the reverse insertion sequence has the strand configuration of $w$ and the number of points is $l(w)$, hence is in $\mathcal{R C}(w)$. Furthermore, a unique $i \leq r$ is given by the final row $i_{p}$.

Remark 5.4. A permutation $w$ is Grassmannian if it has only one descent in the sequence $w_{1}, w_{2}, \ldots$. When $w$ is Grassmannian, the insertion algorithm corresponds to the usual Schensted insertion on semistandard tableaux. We leave it to the reader to check that one can find a weight-preserving bijection between $\mathcal{R C}(w)$ and the set of semistandard tableaux of shape $\lambda(w)$ filled with numbers $1,2, \ldots, r$, where $r$ is the unique descent of $w$. (See [Billey et al. 1993, Theorem 2.5] and [Bergeron 
$1992, \S 2]$.) Then one can show that our insertion algorithm, keeping $r$ fixed, corresponds (under this bijection) to Schensted insertion.

\section{CONJECTURES}

As we know (Corollary 3.9), Schubert polynomials form an integral basis for $\mathbb{Z}\left[x_{1}, x_{2}, \ldots\right]$. A longstanding open problems in the theory of Schubert polynomials is to find a combinatorial proof that the coefficients $c_{u v}^{w}$ are positive in the expansion

$$
\mathfrak{S}_{u} \mathfrak{S}_{v}=\sum c_{u v}^{w} \mathfrak{S}_{w} .
$$

The $c_{u v}^{w}$ are the analogs of the Littlewood-Richardson coefficients in the theory of Schur functions. Monk's rule is a special case of this problem. The two rules conjectured in this Section will be analogs of Pieri's Rule, which was the predecessor of the Littlewood-Richardson rule.

Our investigation of the insertion algorithm in Section 5 suggested a generalization of Monk's rule. We successively insert $b \geq i_{1} \geq i_{2} \geq \cdots \geq i_{d}$ into a diagram. This led us to Conjecture 6.1. We were not able to prove this conjecture by generalizing the proof of Monk's rule, that is, by successive insertions, but we still believe it is true! (Here is a counterexample, found by computer, that shows that successive insertion alone will not prove the conjectures: the multiplication of $\mathfrak{S}_{[1,2,5,4,3]}$ with $\mathfrak{S}_{[1,2,4,5,3]}$. For the insertions of $i_{1}=3$ and $i_{2}=3$ in the rc-graph $D=\{(1,3),(1,4),(2,3)\}$, the second insertion path fails to remain weakly to the right of the first one.)

The second conjecture was found by computer investigation. We assumed that there would be an analogue of Conjecture 6.1 for permutations with diagram $D(w)$ a single column. We programmed several different patterns until a rule emerged. This computation would have been impossible to do by hand because we need to multiply Schubert polynomials and expand in the basis of Schubert polynomials many times for each example.

Let $r[b, d]=[1,2, \ldots, b-1, b+d, b, b+1, \ldots]$. Note that $D_{\text {bot }}(r[b, d])$ is a single row. The per- mutation $r[b, d]$ is Grassmannian, and its Schubert polynomial equals $h_{d}\left(x_{1}, x_{2}, \ldots, x_{b}\right)$, the homogeneous symmetric function of degree $d$.

Conjecture 6.1. For any $w \in S_{\infty}$ and any positive integers $b$ and $d$, we have

$$
\mathfrak{S}_{w} \mathfrak{S}_{r[b, d]}=\sum \mathfrak{S}_{w^{\prime}}
$$

where the sum runs over all $w^{\prime}=w t_{k_{1} l_{1}} t_{k_{2} l_{2}} \ldots t_{k_{d} l_{d}}$ such that $k_{i} \leq b<l_{i}$ for $1 \leq i \leq d$, and if we let $w^{(i)}=w^{(i-1)} t_{k_{i} l_{i}}$ with $w^{(0)}=w$, then $l\left(w^{(i)}\right)=$ $l\left(w^{(i-1)}\right)+1$ and

$$
w_{k_{1}}^{(1)}<w_{k_{2}}^{(2)}<\cdots<w_{k_{d}}^{(d)} .
$$

Remark 6.2. It is remarkable that this multiplication is multiplicity free!

Let $c[b, d]=[1,2, \ldots, b-d, b-d+2, \ldots, b+1$, $b-d+1, b+2, b+3, \ldots]$. Note that $D_{\text {top }}(c[b, d])$ is a single column. The permutation $c[b, d]$ is also Grassmannian, and its Schubert polynomial equals $e_{d}\left(x_{1}, x_{2}, \ldots, x_{b}\right)$, the elementary symmetric function.

Conjecture 6.3. For any $w \in S_{\infty}$ and any positive integers $b$ and $d$, we have

$$
\mathfrak{S}_{w} \mathfrak{S}_{c[b, d]}=\sum \mathfrak{S}_{w^{\prime}}
$$

where the sum runs over all $w^{\prime}=w t_{k_{1} l_{1}} t_{k_{2} l_{2}} \ldots t_{k_{d} l_{d}}$ such that $k_{i} \leq b<l_{i}$ for $1 \leq i \leq d$, and if we let $w^{(i)}=w^{(i-1)} t_{k_{i} l_{i}}$ with $w^{(0)}=w$, then

$$
l\left(w^{(i)}\right)=l\left(w^{(i-1)}\right)+1
$$

and

$$
w_{k_{1}}^{(1)}>w_{k_{2}}^{(2)}>\cdots>w_{k_{d}}^{(d)}>0 .
$$

The conjectures have been verified by computer for all permutations $w \in S_{7}$ and all values of $b$ and $d$. We have found computations in $S_{8}$ to be beyond the capacity of our computers (Sun Sparc 10). The validity of these conjectures would greatly speed up any algorithm for expanding the products $\mathfrak{S}_{w} \mathfrak{S}_{r[b, d]}$ and $\mathfrak{S}_{w} \mathfrak{S}_{c[b, d]}$. To see why this is so, we look at an example. 
Suppose that we want to expand $\mathfrak{S}_{r[3,2]} \mathfrak{S}_{[1,2,5,4,3]}$ in the basis of Schubert polynomials. Let $b=3$ and $d=2$, and construct a rooted tree in the following way:

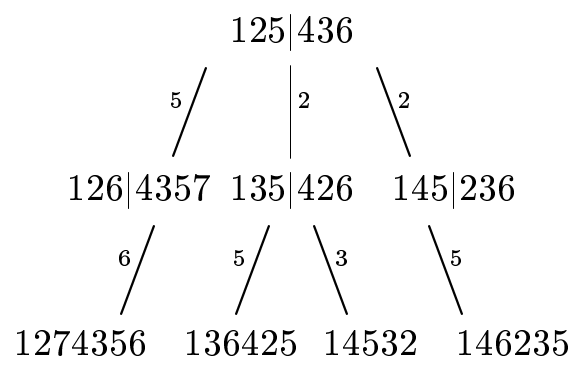

The top of the tree is the initial permutation. We assume there are an infinite number of fixed points beyond what is written. We have inserted a vertical line after the position $b=3$. To find the children of the root, we find all transpositions that switch numbers across the vertical line so that the lengths increase by exactly one. We label the edge from the root to a child by the smallest of the two numbers switched. Of course the smallest number will always come from the left. This constructs the first generation of the tree. For the next generation, we repeat the process above, but only allowing the transpositions for which the smallest number is bigger than the label on the edge of this node. We repeat the last step $d=2$ times. The leaves of the tree are precisely the permutations $w^{\prime}$ that appear in the expansion in Conjecture 6.1.

\section{ACKNOWLEDGEMENTS}

We would like to thank several people for valuable suggestions, in particular Sergey Fomin, Adriano Garsia, Mark Haiman, Arun Ram, Richard Stanley, and Glenn Tesler.

\section{REFERENCES}

[Bergeron 1992] N. Bergeron, "A combinatorial construction of the Schubert polynomials", J. Comb. Theory A60 (1992), 168-182.

[Bernstein et al. 1973] I. N. Bernstein, I. M. Gelfand and S. I. Gelfand, "Schubert cells and cohomology of the spaces G/P", Russian Math. Surveys 28 (1973), $1-26$.

[Billey et al. 1993] S. Billey, W. Jockusch and R. Stanley, "Some Combinatorial Properties of Schubert Polynomials", J. Alg. Comb. (2) 4 (1993), 345-374.

[Demazure 1974] M. Demazure, "Désingularization des variétés de Schubert généralisées", Ann. Sci. Éc. Norm. Sup. (Paris) 7 (1974) 53-58.

[Fomin and Kirillov 1993] S. Fomin and A. N. Kirillov, "Yang-Baxter equation, symmetric functions, and Schubert polynomials", submitted to the Proceedings of the Conference on Power Series and Algebraic Combinatorics, Firenze, 1993.

[Fomin and Stanley 1991] S. Fomin and R. Stanley, "Schubert Polynomials and the NilCoxeter algebra", Adv. Math. 103 (1994), 196-207.

[Kohnert 1990] A. Kohnert, "Weintrauben, Polynome, Tableaux", Bayreuth. Math. Schrift. 38 (1990), 1-97.

[Lascoux and Schützenberger 1982] A. Lascoux and M.P. Schützenberger, "Polynômes de Schubert", C. R. Acad. Sci. Paris 294 (1982) 447-450.

[Lascoux and Schützenberger 1985] A. Lascoux and M.P. Schützenberger, "Schubert polynomials and the Littlewood-Richardson Rule", Let. Math. Phys. 10 (1985) 111-124.

[Macdonald 1991] I. G. Macdonald, Notes on Schubert Polynomials, Publications du LACIM 6, Université du Québec à Montréal (1991).

[Schubert 1879] H. Schubert, Kalkül der Abzählenden Geometrie, Teubner, Leipzig, 1879.

Nantel Bergeron, Department of Mathematics, Harvard University, Cambridge, MA 02138 (nantel@math.harvard.edu)

Sara Billey, Department of Mathematics, University of California at San Diego, La Jolla, CA 92093-0112 (sara@macaulay.ucsd.edu)

Received October 29, 1993; accepted in revised form January 15, 1994 